\title{
Clinical Features of Recurrence in Patients Without Residual Tumour in Endometrial Cancer
}

\author{
DAISUKE MIYAHARA ${ }^{1}$, FUSANORI YOTSUMOTO ${ }^{1}$, TOYOFUMI HIRAKAWA ${ }^{1}$, KENICHI YOSHIKAWA ${ }^{1}$, \\ KOICHIRO SHIGEKAWA ${ }^{1}$, KOHEI MIYATA ${ }^{1}$, TOMOHIRO ITO ${ }^{1}$, SUNG OUK NAM $^{1}$, \\ MASAMITSU KURAKAZU ${ }^{1}$, YASUNOBU KANAMORI ${ }^{2}$, SATOSHI AMADA ${ }^{3}$ and SHINGO MIYAMOTO ${ }^{1}$ \\ ${ }^{1}$ Department of Obstetrics and Gynecology, Faculty of Medicine, Fukuoka University, Fukuoka, Japan; \\ ${ }^{2}$ Department of Obstetrics and Gynecology, Japanese Red Cross Yamaguchi Hospital, Yamaguchi, Japan; \\ ${ }^{3}$ Department of Obstetrics and Gynecology, Kitakyushu Municipal Medical Center, Kitakyushu, Japan
}

\begin{abstract}
Background/Aim: Initial treatment of endometrial cancer with surgery and platinum and taxane-based chemotherapy is often successful, but it remains unclear as to whether certain types of the disease relapse. The aim of this study was to identify the clinical features of recurrence in patients without residual tumour in endometrial cancer. Patients and Methods: Clinical features, histological type, and time to recurrence were analyzed in 640 endometrial cancer patients without residual tumours. Results: Of 640 patients, 517 were type I and 123 were type II. For type I, early recurrent $(E R)$ disease and late recurrent $(L R)$ disease were noted in 80 and 8 patients, respectively, and $97.5 \%$ of $E R$ occurred within 2 years. After recurrence, $76.2 \%$ of ER and $50 \%$ of LR patients died. In type II, ER and LR were noted in 41 and 1 patients, respectively, and $97.6 \%$ of ER occurred within 2 years, of which $75.6 \%$ died after recurrence. One LR case died of disease. Conclusion: Most patients recurred within 2 years irrespective of clinical stage or type.
\end{abstract}

The incidence of endometrial cancer is increasing year upon year. Endometrial cancer (EC) occurs at a higher rate than cervical cancer, ovarian cancer, and vaginal cancer, and is the most common malignancy of the female reproductive tract in Japan and other high-income countries. In 2012, 76,000 deaths worldwide were due to EC (1). Thus, improvement in EC prognosis is required on a global scale. Treatment for EC mainly consists of cytoreductive surgery

Correspondence to: Shingo Miyamoto, MD, Ph.D., Department of Obstetrics and Gynecology, Faculty of Medicine, Fukuoka University, 7-45-1 Nanakuma, Jonan-ku, Fukuoka 814-0180, Japan. Tel: +81 928011011, Fax: +81928654114,e-mail: smiya@ cis.fukuoka-u.ac.jp

Key Words: Endometrial cancer, late recurrence, Type I, Type II. and adjuvant chemotherapy. In the early stages of EC, cytoreductive surgery is the standard of care, while cytoreductive surgery and adjuvant chemotherapy including platinum and taxanes are used for advanced EC. In general, EC is successfully treated by the first round of treatment. However, the majority for patients with advanced EC recur within a median period of 3 years and $15-20 \%$ of recurrence is observed even in patients without residual disease (2-4). Many recurrence sites are observed; local recurrence to the vaginal cuff, or distant metastasis to lungs. However, late recurrence at more than 3 years is not well understood in patients with EC.

EC is pathologically classified into type I and type II. Type I EC, which is an oestrogen-related tumour, accounts for approximately $80 \%$ of EC cases. In addition, the histology of type I EC is regarded as grade 1 or grade 2 endometrioid adenocarcinoma and mucinous adenocarcinoma, according to the Japan Society of Gynaecologic Oncology (JSGO) guidelines. Type II EC, which is thought to be an oestrogennon-related tumour, accounts for the remaining 15\%-20\% of EC cases. The histology of type II EC, which contributes to its heterogeneity, includes serous, clear cell adenocarcinoma, grade 3 endometrioid adenocarcinoma and other types of adenocarcinoma. Patients with type I EC, generally, have a more favourable prognosis compared with patients with type II EC (5). In the 2015 JSGO Annual Report, 48.7\%, 20.3\%, $8.5 \%, 5.4 \%, 2.2 \%$, and $14.9 \%$ of diagnosed patients had endometrioid adenocarcinoma grade $1(\mathrm{G} 1)$, grade $2(\mathrm{G} 2)$, grade 3 (G3), serous adenocarcinoma, clear cell carcinoma, and other disease, respectively. The 5-year survival by histological type for patients with ECs was $95.4 \%$ for G1 endometrioid adenocarcinoma, $87.3 \%$ for G2 endometrioid adenocarcinoma, $71.0 \%$ for G3 endometrioid adenocarcinoma, $57.1 \%$ for serous adenocarcinoma, and $65.3 \%$ for clear cell carcinoma (6). However, few reports have examined the differences in recurrence time or site between type I and type II EC. 
To investigate the prognosis of EC, the differences in recurrence time between type I and type II endometrial cancer at each clinical stage in patients without residual tumours after treatment we compared and the recurrence time or sites over 3 years after successful treatment of this disease were assessed.

\section{Materials and Methods}

This study was a joint project of the Fukuoka University Hospital, the Kitakyushu Municipal Medical Centre, and the Japanese Red Cross Yamaguchi Hospital. Initial treatment and recurrence treatment for EC patients was performed at each hospital. The clinical, surgical, and pathological data of the patients were collected from patient files. Patients with unknown grade/stage, non-surgical primary therapy, carcinosarcoma, and multiple primary neoplasm, and a follow-up period of less than 30 days were excluded. All patient data were evaluated retrospectively.

Six-hundred and forty patients with newly-diagnosed EC were enrolled between January 2003 and December 2012. This retrospective investigation assessed patients who underwent peritoneal washing, total hysterectomy or radical hysterectomy, bilateral salpingo-oophorectomy, pelvic lymphadenectomy, and para-aortic lymph node dissection for EC. No patients underwent surgical resection with a laparoscopic or robotic procedure.

We reclassified the 640 patients according to the guidelines for EC determined by the International Federation of Gynecology and Obstetrics (FIGO) in 2008. Risk of EC recurrence was based on 2013 guidelines set by the JSGO. The treatment provided to lowrisk patients was surgery alone, while intermediate-risk and highrisk patients also received anticancer agents. Anticancer agents were continued until disease progression or treatment completion. Treatment in the intermediate-risk group comprised three cycles, and treatment in the high-risk group comprised six cycles. Patients received cyclophosphamide plus doxorubicin plus cisplatin (CAP) from January 2003 to August 2005 and DP after September 2005. CAP administration comprised cyclophosphamide $\left(333 \mathrm{mg} / \mathrm{m}^{2}\right)$, doxorubicin $\left(40 \mathrm{mg} / \mathrm{m}^{2}\right)$, and cisplatin $\left(50 \mathrm{mg} / \mathrm{m}^{2}\right)$ every 28 days. DP administration comprised docetaxel $\left(70 \mathrm{mg} / \mathrm{m}^{2}\right)$ and cisplatin $\left(60 \mathrm{mg} / \mathrm{m}^{2}\right)$ every 28 days. There were cases in which irinotecan plus cisplatin (CPT-11/CDDP) was administered for clear cell carcinoma. CPT-11/CDDP administration comprised irinotecan (60 $\mathrm{mg} / \mathrm{m}^{2}$ on day 1 , day 8 , and day 15$)$ and cisplatin $\left(60 \mathrm{mg} / \mathrm{m}^{2}\right.$ on day 1) every 28 days.

Routine follow-up at intervals of 1-3 months for 1-3 years, and every 6 months for the fourth and fifth years was performed. Follow-up was not performed after the fifth year. Routine followup after initial treatment included a combination of pelvic examination, vaginal cytology, transvaginal ultrasonography, tumour marker measurements, and CT scanning (every 6 months or every 12 months).

Time to recurrence or progression was measured from the date of the last treatment to the date of the documented event. Patients were classified into three categories: 1) non-recurrent (NR); 2) early recurrent $(\mathrm{ER}),<5$ years between the end of primary therapy and recurrence; and 3) late recurrent (LR), $>5$ years between the end of primary therapy and the appearance of 'recurrent' disease. Time until recurrence, the site of recurrence, recurrence treatment, and type of EC (e.g. type I, type II) were analysed.
Table I. Clinical characteristics of patients.

\begin{tabular}{lccc}
\hline & $\begin{array}{c}\mathrm{NR} \\
(\mathrm{n}=510)\end{array}$ & $\begin{array}{c}\text { ER } \\
(\mathrm{n}=121)\end{array}$ & $\begin{array}{c}\mathrm{LR} \\
(\mathrm{n}=9)\end{array}$ \\
\hline $\begin{array}{l}\text { Age (years, median [range] }) \\
\text { Performance status (n) }\end{array}$ & $58[29-91]$ & $62[38-87]$ & 53 [34-67] \\
0 & 510 & 121 & 9 \\
Histology (n) & & & \\
Endometrioid adenocarcinoma & & & \\
G1 & 297 & 55 & 4 \\
G2 & 132 & 25 & 4 \\
G3 & 63 & 14 & 0 \\
Serous adenocarcinoma & 3 & 15 & 1 \\
Clear cell adenocarcinoma & 6 & 6 & 0 \\
Mucinous adenocarcinoma & 6 & 0 & 0 \\
Others & 3 & 6 & 0 \\
Stage (FIGO 2008) (n) & & & \\
IA/IB & $276 / 66$ & $41 / 7$ & $1 / 0$ \\
II & 30 & 15 & 2 \\
IIIA/IIIB/IIIC & $66 / 6 / 60$ & $3 / 0 / 13$ & $2 / 1 / 0$ \\
IVA/IVB & $0 / 6$ & $3 / 39$ & $3 / 0$ \\
\hline
\end{tabular}

NR: Non-recurrence; ER: early recurrence; LR: late recurrence.

\section{Results}

Patient characteristics. Patient characteristics are shown in Table I. Five years or more after the initial successful management, there were $510 \mathrm{NR}$ patients, 121 ER patients, and nine LR patients remaining in the study. All patients had an Eastern Cooperative Oncology Group performance status of zero.

The median age in the NR group at diagnosis was 58 years (range $=29-91$ years). According to the histological subtype, type I EC was present in $84.1 \%$ (429/510) of patients and type II EC was present in $15.9 \%(81 / 510)$ of patients. The FIGO (2008) classifications in the NR group were as follows: 342 patients $(67 \%)$ had stage I, 30 patients (5.9\%) had stage II, 132 patients $(25.9 \%)$ had stage III, and six patients $(1.2 \%)$ had stage IV disease. In stage I patients, the type of adjuvant treatment after primary surgery was CAP in 12 patients (type I, three patients; type II, nine patients) and DP in 69 patients (type I, 39 patients; type II, 30 patients). In stage II patients, the type of adjuvant treatment after primary surgery was CAP in three patients (type I, three patients) and DP in 15 patients (type I, nine patients; type II, six patients). The other patients were all type I, and after treatment, adjuvant treatment was discontinued at the patient's discretion. In stage III patients, the type of adjuvant treatment after primary surgery was CAP in 36 patients (type I, 33 patients; type II, three patients), DP in 63 patients (type I, 51 patients; type II, 12 patients), and CPT-11/CDDP in three patients (type II, three 

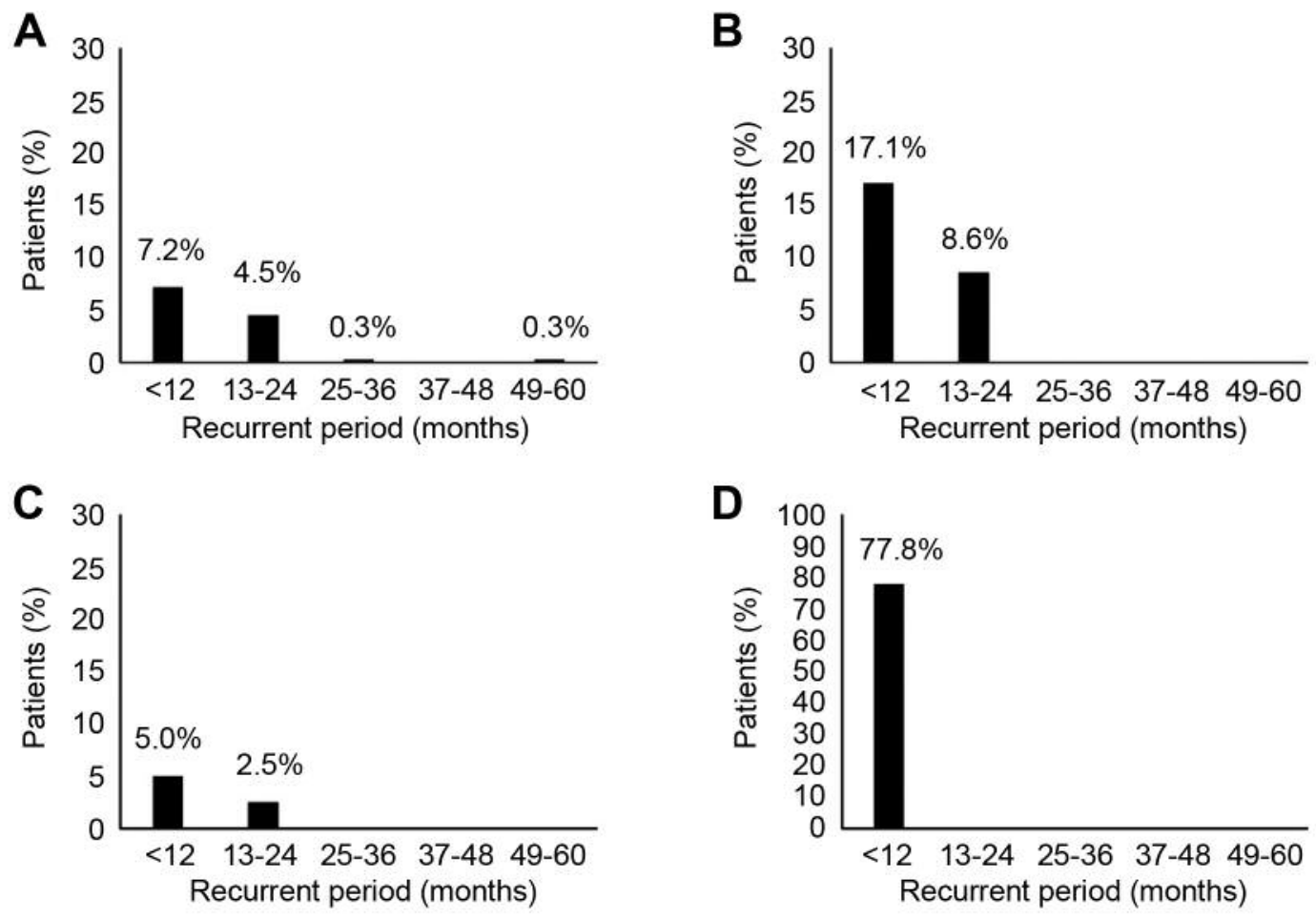

Figure 1. Frequency distribution of recurrence periods in early-recurrent patients with type I endometrial cancer. (A) Stage I ( $n=41)$, (B) stage II $(n=9),(C)$ stage III $(n=9)$, and (D) stage IV $(n=21)$.

patients). Thirty-three patients did not receive adjuvant treatment due to abdominal abscess, old age, or Alzheimer's dementia. In stage IV patients, CAP was administered to three patients (type II, three patients) and DP was administered to three patients (type I, three patients).

The median age of the ER group at diagnosis was 62 years (range $=38-87$ years). According to the histological subtype, type I EC was present in $66.1 \%(80 / 121)$ of patients and type II EC was present in $33.9 \%$ (41/121) of patients. The FIGO (2008) classifications in the ER group were as follows: 48 patients had stage I, 15 patients had stage II, 16 patients had stage III, and 42 patients had stage IV disease. In stage I patients, the type of adjuvant treatment after primary surgery was DP in eight patients (type I, seven patients; type II, one patient), and CPT$11 /$ CDDP in three patients (type II, three patients). In stage II patients, the type of adjuvant treatment after primary surgery was CAP in three patients (type I, three patients) and DP in 12 patients (type I, six patients; type II, six patients). In stage III patients, the type of adjuvant treatment after primary surgery was CAP in three patients (type I, three patients) and DP in 13 patients (type I, six patients; type II, seven patients). In stage IV patients, DP was administered to 33 patients (type I, 18 patients; type II, 15 patients) and CPT-11/CDDP was administered to three patients (type II, three patients). In six patients, adjuvant treatment could not be administered because of the general condition of the patient after surgery.

The median age of the LR group at diagnosis was 53 years (range=34-67 years). According to the histological subtype, type I EC was present in $88.9 \%$ (8/9) patients and type II EC was present in $11.1 \%$ (1/9) of patients. The FIGO (2008) classifications in the LR group were as follows: one patient had stage I, two patients had stage II, three patients had stage III, and three patients had stage IV disease. In stage I patients, the type of adjuvant treatment after primary surgery was DP in one patient (type II, one patient). In stage II patients, the type of adjuvant treatment after primary surgery was DP in two patients (type I, two patients). In stage III patients, the type of adjuvant treatment after primary surgery was CAP in one patient (type I, one patient) and DP in two patients (type I, two patients). In stage IV patients, the type of adjuvant treatment after primary surgery was CAP in two patients (type I, two patients) and DP in one patient (type I, one patient).

All patients completed the treatment with total remission, as determined by clinical medical examination, markers (CA125<35 UI/ml), and imaging (CT, ultrasound, chest $\mathrm{X}$-ray). Routine follow-up was performed after initial treatment. 

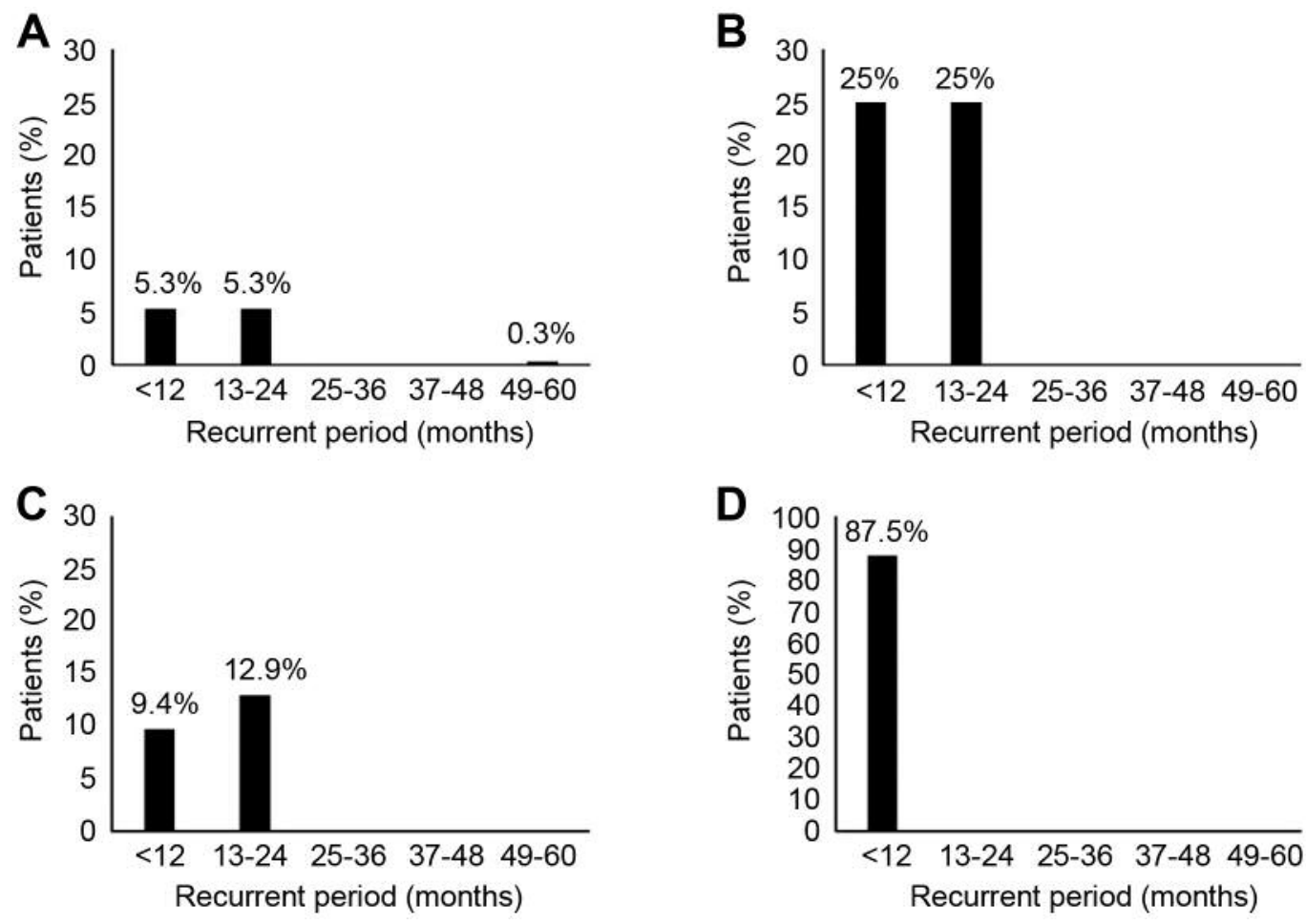

Figure 2. Frequency distribution of recurrence periods in early-recurrent patients with type II endometrial cancer. (A) Stage I ( $n=7)$, (B) stage II $(n=6),(C)$ stage III $(n=7)$, and (D) stage IV $(n=21)$.

Clinical characteristics of the ER group and outcomes. There were 121 patients in the ER group and the median time to ER was 9 months (range $=1-57$ months). Figures 1 and 2 show the recurrence periods based on initial diagnosis (type I EC or type II EC) and stage of the disease (stage I, II, III, or IV).

Among patients with an initial diagnosis of type I EC, 335 were stage I, and ER occurred in 41 patients. Of these patients, ER occurred within 12 months in 24 (7.2\%) patients, between 12 and 24 months in $15(4.5 \%)$ patients, between 24 and 32 months in one $(0.3 \%)$ patient, and at 58 months in one $(0.3 \%)$ patient (Figure 1A).

The patient who experienced recurrence at 32 months was a 65-year-old woman who was diagnosed with EC stage IA, endometrioid adenocarcinoma grade 2 , and was treated with primary surgery. No adjuvant therapy was administered because this case was considered low-risk. The patient remained under close follow-up and showed no evidence of recurrent disease until 31 months. The CT scan at 32 months showed positive para aortic lymph node metastasis and the patient was treated with brachytherapy to the upper para aortic lymph nodes with a dose of 45 Gray. This patient remained free of recurrent or persistent disease for more than 5 years after salvage treatment.
Table II. Comparison of relapse sites in the early-recurrence group.

\begin{tabular}{lcc}
\hline Relapse sites & $\begin{array}{c}\text { Type I }(\mathrm{n}=80) \\
\text { (stage I/II/III/IV) }\end{array}$ & $\begin{array}{c}\text { Type II }(\mathrm{n}=41) \\
\text { (stage I/II/III/IV) }\end{array}$ \\
\hline Peritoneal dissemination & $40(25 / 3 / 3 / 9)$ & $13(1 / 0 / 0 / 12)$ \\
Para-aortic lymph nodes & $19(7 / 3 / 3 / 6)$ & $9(3 / 0 / 3 / 3)$ \\
Lung & $18(9 / 0 / 3 / 6)$ & $12(3 / 6 / 0 / 3)$ \\
Pelvic lymph nodes & $3(0 / 3 / 0 / 0)$ & $3(0 / 0 / 3 / 0)$ \\
Bone & 0 & $3(0 / 0 / 0 / 3)$ \\
Pleurisy & 0 & $1(0 / 0 / 1 / 0)$ \\
\hline
\end{tabular}

The patient who experienced recurrence at 58 months was a 58-year-old woman who was diagnosed with EC stage IB, endometrioid adenocarcinoma grade 1 , and was treated with primary surgery. She was treated with DP as she was considered as intermediate-risk. The CT scan at 58 months was positive for peritoneal dissemination and pelvic lymph node metastasis and the patient was treated with AP. She remained free of recurrent or persistent disease for more than 5 years after salvage treatment.

There were 35 type I EC patients with an initial diagnosis of stage II disease and ER occurred in nine of 
Table III. Patients' characteristics and outcome of initial treatment.

\begin{tabular}{lcccc}
\hline Case & Age & FIGO stage & Histology & Initial treatment \\
\hline 1 & 67 & IA & Serous & TH + BSO + PPLND + OM $\rightarrow$ DC \\
2 & 53 & IIB & Endometrioid G1 & mRTH + BSO + PPLND $\rightarrow$ DP \\
3 & 60 & IIB & Endometrioid G1 & RTH + BSO + PPLND $\rightarrow$ DP \\
4 & 52 & IIIA & Endometrioid G1 & mRTH + BSO + PPLND $\rightarrow$ CAP \\
5 & 56 & IIIA & Endometrioid G1 & RTH + BSO + PPLND + OM $\rightarrow$ DC \\
6 & 58 & IIIC & Endometrioid G2 & TH + BSO + PPLND + OM $\rightarrow$ DP \\
7 & 35 & IVB & Endometrioid G2 & TH + BSO + PPLND + OM $\rightarrow$ CAP \\
8 & 44 & IVB & Endometrioid G2 & mRTH + BSO + PPLND + OM $\rightarrow$ AP \\
9 & 39 & IVB &
\end{tabular}

RTH: Radical hysterectomy; mRTH: modified radical hysterectomy; TH: total hysterectomy; BSO: bilateral salpingo-oophorectomy; PPLND: pelvic and para-aortic lymph node dissections; OM: omentectomy; CAP: cisplatin-adriamycin-cyclophosphamide; DP: docetaxel-cisplatin; AP: adriamycincisplatin; DC: docetaxel-carboplatin.

those patients. ER occurred within 12 months in six $(17.1 \%)$ patients and between 12 and 24 months in three $(8.6 \%)$ patients (Figure 1B).

There were 120 type I patients with a diagnosis of stage III disease, and ER occurred in nine patients. Of these patients, ER occurred within 12 months in six (5.0\%) patients and between 12 and 24 months in three $(2.5 \%)$ patients (Figure 1C).

There were 27 type I patients with an initial diagnosis of stage IV, and ER occurred in 21 of these patients. Among them, ER occurred within 12 months in $21(77.8 \%)$ patients (Figure 1D). All 21 patients died from recurrent EC. In the six patients who did not experience ER, three patients had lesions in the peritoneum and omentum, which were removed during the initial surgery, and the patients were treated with DP. The other three patients experienced LR that occurred after 5 years.

There were 56 type II patients with an initial diagnosis of stage I and ER occurred in seven of those patients. ER occurred within 12 months in three $(5.3 \%)$ patients, between 12 to 24 months in three $(5.3 \%)$ patients, and at 57 months in one $(0.3 \%)$ patient (Figure 2A). The patient who experienced ER at 57 months was a 40-year-old woman who was diagnosed with EC stage IA, endometrioid adenocarcinoma grade 3 , and treated with total hysterectomy, bilateral salpingo-oophorectomy, and pelvic and para-aortic lymph node dissection. She was treated with DP because she was considered as intermediate-risk. The CT scan at 57 months was positive for peritoneal dissemination, and at the time of this study, the patient was receiving weekly paclitaxel and carboplatin therapy.

There were 12 type II patients with an initial diagnosis of stage II and ER occurred in six of those patients. ER occurred within 12 months in three (25\%) patients and between 12 and 24 months in three $(25 \%)$ patients (Figure $2 \mathrm{~B}$ ).
There were 31 type II patients with an initial diagnosis of stage III and ER occurred in seven of those patients. ER occurred within 12 months in three $(9.4 \%)$ patients and between 12 and 24 months in four (12.9\%) patients (Figure 2C).

There were 24 type II patients with an initial diagnosis of stage IV and ER occurred in 21 of those patients. ER occurred within 12 months in $21(87.5 \%)$ patients (Figure 2D). All 21 patients died from recurrent EC.

Figures 1 and 2 show that the majority of ER for both type I and type II disease occurred within 24 months.

Table II shows the organs involved in ER in type I and type II patients. For both type I and type II, recurrence sites included the peritoneum, para-aortic lymph nodes, and lungs. The peritoneum was the most common recurrence site with $50 \%$ (40 cases) in type I and $31 \%$ (13 cases) in type II. In type I EC, $62.5 \%$ were stage I, whilst in type II EC, $92.3 \%$ were stage IV, confirming that in type II EC, the disease stage was more severe. The prevalence of paraaortic lymph node involvement did not differ for type I and type II EC. Bone metastasis and pleurisy were confirmed only in type II EC.

In the type I group, according to recurrent treatment analyses, 43 patients (stage I, 31 patients; stage III, three patients; stage IV, nine patients) underwent chemotherapy, 22 patients (stage I, 10 patients; stage II, nine patients; stage III, three patients) underwent radiotherapy, and 15 patients (stage III, three patients; stage IV, 12 patients) received best supportive care.

In the type II group, according to recurrent treatment analyses, 23 patients (stage I, seven patients; stage II, six patients; stage III, one patient; stage IV, nine patients) underwent chemotherapy, six patients (stage III, six patients) underwent radiotherapy, and 12 patients (stage IV, 12 patients) received best supportive care. 
Table IV. Clinical characteristics of late relapse and outcome.

\begin{tabular}{lcccccc}
\hline Case & $\begin{array}{c}\text { Time to relapse } \\
\text { (month) }\end{array}$ & $\begin{array}{c}\text { Opportunity of } \\
\text { the diagnosis }\end{array}$ & $\begin{array}{c}\text { CA125 } \\
(\mathrm{IU} / \mathrm{ml})\end{array}$ & $\begin{array}{c}\text { Tumor } \\
\text { site }\end{array}$ & $\begin{array}{c}\text { Treatment after } \\
\text { recurrence }\end{array}$ & $\begin{array}{c}\text { Outcome } \\
\text { (follow-up, months) }\end{array}$ \\
\hline 1 & 127 & Abdominal pain & 2749 & PM & TC & DOD (3) \\
2 & 70 & Physical examination & 8 & Vaginal stump & SDS, DP & NED (23) \\
3 & 77 & AGB & 11 & Vaginal stump & RT, TC & NED (72) \\
4 & 62 & Abdominal pain & 53 & PM, PLN & AP & DOD (12) \\
5 & 104 & Abdominal pain & ND & PM & SDS, TC, DC & DOD (69) \\
6 & 78 & No symptom & 9.9 & PAN & RT & DOD (25) \\
7 & 84 & Abdominal pain & 25 & PM & SDS, DP & NED (3) \\
8 & 233 & Cough & 10 & lung & NATS & RT, TC \\
9 & 70 & Oliguria & 60 & Iliopsoas muscle, PAM & DOD (18) \\
\hline
\end{tabular}

ABG: Atypical genital bleeding; PAN: para aortic lymph node; PM: pelvic mass; SDS: secondary debulking surgery; VATS: video-assisted thoracic surgery; TC: paclitaxel-carboplatin; RT: radiation therapy; CAP: cisplatin-adriamycin-cyclophosphamide; DP: docetaxel-cisplatin; AP: adriamycincisplatin; DC: docetaxel-carboplatin; NED: no evidence of disease; DOD: died of disease; ND: not determined.

Clinical characteristics of the LR group and outcomes. Tables III and IV list the characteristics of the LR group and outcomes of the treatment that was subsequently performed after LR. Eight patients were diagnosed with type I endometrioid carcinoma (grade I, four patients; grade II, four patients) and one patient had serous carcinoma. Median time to LR was 77 months (range=62-233 months). Nine patients presented with various symptoms, including abdominal pain (four patients), atypical genital bleeding (one patient), and oliguria (one patient). Three patients were asymptomatic. In all patients, the sites of recurrence were determined by clinical and radiological examinations. Salvage chemotherapy was performed at recurrence in patients 2,5 , and 7 , following reduction surgery. At the time of this study, patient 2 and 7 remain alive. Patient 5 died of cancer 69 months after recurrence. In patient 8 , for whom video-assisted-thoracicsurgery (VATS) was performed, no salvage chemotherapy was administered. Patient 8 is also alive following recurrence.

Two patients underwent cisplatin-based chemotherapy. However, the cisplatin-based chemotherapy after recurrence did not have the therapeutic effect of chemotherapy. Two patients died. Three patients underwent radiotherapy plus cisplatin-based chemotherapy, and of these, two patients died from their disease and one patient remains alive with the disease.

\section{Discussion}

In this study, all patients with type II EC relapsed within 12 months for stage IV disease and within 24 months for stage I, II and III, except for two patients with stage I. Most patients with type I EC also relapsed within 24 months at stage I, II, III, and IV. A few patients with type I EC relapsed after 48 months for each clinical stage. Nine out of 640 patients with EC (1.4\%) relapsed after 5 years.
Smith et al. have reported that recurrence occurred at $>5$ years in approximately $20 \%$ of patients with type I EC after initial treatment and that there were very few patients with recurrence at more than 10 years after the initial treatment, suggesting that follow-up is recommended within 10 years of initial treatment (7). In patients with recurrence at more than 10 years, time to recurrence and recurrent organ was 26.5 years at vaginal sites, 14 years at abdominal operation scar, 11 years at the upper urinary tract, and 17 years at vaginal sites (8-11). In these four patients, the histological subtype was type I and the clinical stages included stage I, II, or III. In this study, most patients with type I EC recurred within 24 months and the incidence of recurrence decreased even more than before, suggesting that adjuvant chemotherapy may contribute to anti-tumour effects in type I EC.

Analysis of the clinicopathological features revealed that type II EC is generally more aggressive than type I EC and is associated with a poorer prognosis even when diagnosed at an early stage (12). In this study, the majority of patients with type II EC relapsed within 24 months. Only two patients recurred at 57 months and 127 months later after the initial treatment, and their histological subtype displayed serous adenocarcinoma. In addition, these two patients presented diffuse abdominal peritoneal dissemination, which was confirmed by CT scan at recurrence. Both patients died within 12 months even though they received chemotherapy including carboplatin and paclitaxel. However, it is thought that they had an onset of peritoneal cancer and not a recurrence endometrial cancer. According to this evidence, patients with type II EC would relapse within 24 months if they have recurred.

The development of novel targeted agents is urgently required for EC. The recent genotyping of EC is likely to represent a milestone in the development of novel therapeutic strategies. As a result, the mapping of the genomic landscape of EC has identified four molecular subgroups: 1) POLE ultra-mutated; 2) 
MSI-hypermutated; 3) copy-number low endometrioid; and 4) copy-number high serous-like (13). The POLE ultra-mutated and MSI-hypermutated groups are characterized by an active immune microenvironment as demonstrated by the abundance of tumour-specific neo-antigens and a high number of tumourinfiltrating lymphocytes (TILs), leading to overexpression of PD1 and PD-L1 (14). In addition, immune checkpoints such as CTLA-4, LAG-3, and IDO may also be upregulated in POLE ultra-mutated and MSI-hypermutated EC (14-16). In other reports, PD-1, PD-L1, and PD-L2 expression in tumourinfiltrating immune cells was more frequently found in patients with type II EC compared with patients with type I EC (17). Accordingly, treatment of the subgroups with frequent expression of the PD-1/PD-L1/PD-L2 axis with anti-PD-1 or anti-PD-L1 antibody-based therapies would induce favourable outcomes in patients with advanced and recurrent EC.

In conclusion, most patients with EC recurred within 2 years, irrespective of clinical stage and type. Especially, in type II EC, the prognosis was extremely poor. In addition, patients with recurrent endometrial cancer except for local recurrence such as that to the vaginal cuff also indicated an unfavourable outcome regardless of type. In POLE ultramutated and MSI-hypermutated EC, up-regulation of the PD-1/PD-L1/PD-L2 axis was observed, suggesting a rationale for testing $\mathrm{PD}-1 / \mathrm{PD}-\mathrm{L} 1$ immunotherapy in patients with advanced and recurrent EC.

\section{Funding}

This work was supported, in part, by JSPS KAKENHI Grant Number 18K09242 and a Grant-in-Aid from the Kakihara Science and Technology Foundation (Fukuoka, Japan) to S. Miyamoto.

\section{Conflicts of Interest}

No potential conflicts of interest exist regarding this study.

\section{Authors' Contributions}

Conception and design of the study: Daisuke Miyahara and Shingo Miyamoto; Analysis and interpretation of data: Daisuke Miyahara, Fusanori Yotsumoto, Toyofumi Hirakawa, Kenichi Yoshikawa, Koichiro Shigekawa, Kohei Miyata, Tomohiro Ito, Sung Ouk Nam, Masamitsu Kurakazu, Yasunobu Kanamori and Satoshi Amada; Collection and assembly of data: Daisuke Miyahara, Yasunobu Kanamori and Satoshi Amada; Drafting of the article: Daisuke Miyahara and Shingo Miyamoto; Critical revision of the article for important intellectual content: Daisuke Miyahara; Final approval of the article: Shingo Miyamoto.

\section{Acknowledgements}

The Authors would like to thank H. Nikki March, PhD, from Edanz Group (www.edanzediting.com/ac) for editing a draft of this manuscript.

\section{References}

1 Ferlay J, Soerjomataram I, Dikshit R, Eser S, Mathers C, Rebelo M, Parkin DM, Forman D and Bray F: Cancer incidence and mortality worldwide: Sources, methods and major patterns in GLOBOCAN 2012. Int J Cancer 136(5): E359-86, 2015. PMID: 2520842. DOI: $10.1002 / \mathrm{ijc} .29210$

2 Fung-Kee-Fung M, Dodge J, Elit L, Lukka H, Chambers A and Oliver T: Follow-up after primary therapy for endometrial cancer: a systematic review. Gynecol Oncol 101(3): 520-529, 2006. PMID: 16556457. DOI: 10.1016/j.ygyno.2006.02.011

3 Creutzberg CL, van Putten WL, Koper PC, Lybeert ML, Jobsen JJ, Wárlám-Rodenhuis CC, De Winter KA, Lutgens LC, van den Bergh AC, van der Steen-Banasik E, Beerman $\mathrm{H}$ and van Lent M: Survival after relapse in patients with endometrial cancer: results from a randomized trial. Gynecol Oncol 89(2): 201-209, 2003. PMID: 12713981.

4 Sohaib SA, Houghton SL, Meroni R, Rockall AG, Blake P and Reznek RH: Recurrent endometrial cancer: patterns of recurrent disease and assessment of prognosis. Clin Radiol 62(1): 28-34, 2007. PMID: 17145260. DOI: 10.1016/j.crad.2006.06.015

5 Bokhman J: Two pathogenetic types of endometrial carcinoma. Gynecol Oncol 15(1): 10-17, 1983. PMID: 6822361.

6 Nagase S, Ohta T, Takahashi F and Enomoto T: The 2017 Committee on Gynecologic Oncology of the Japan Society of Obstetrics and Gynecology: Annual report of the committee on gynecologic oncology, the Japan Society of Obstetrics and Gynecology: Annual patients report for 2015 and annual treatment report. J Obstet Gynaecol Res 45(2): 289-298, 2019. PMID: 30426591. DOI: 10.1111/jog.13863

7 Smith CJ, Heeren M, Nicklin JL, Perrin LC, Land R, Crandon $\mathrm{AJ}$ and Obermair A: Efficacy of routine follow-up in patients with recurrent uterine cancer. Gynecol Oncol 107(1): 124-129, 2007. PMID: 17655917. DOI: 10.1016/j.ygyno.2007.06.002

8 Lederman GS, Niloff JM, Redline R and Rosen EM: Late recurrence in endometrial carcinoma. Cancer 59(4): 825-828, 1987. PMID: 3802041. DOI: 10.1002/1097-0142(19870215)59:4 $<825$ ::aid-cncr2820590428>3.0.co;2-9

9 Lorenz U, Gassel AM, Thiede A and Gassel HJ: Endometrial carcinoma recurrence in an abdominal scar 14 years after total hysterectomy. Gynecol Oncol 95(2): 393-395, 2004. PMID: 15491763. DOI: 10.1016/j.ygyno.2004.05.043

10 Tsurumaki Y, Kume H and Homma Y: Late recurrence of uterine endometrioid carcinoma in the upper urinary tract. Arch Gynecol Obstet 280(4): 631-632, 2009. PMID: 19198862. DOI: 10.1007/ s00404-009-0949-8

11 Yechieli R, Robbins JR, Schultz D, Munkarah A and Elshaikh MA: Vaginal recurrence more than 17 years after hysterectomy and adjuvant treatment for uterine carcinoma with successful salvage brachytherapy: a case report. Case Rep Oncol 4(1): 242-245, 2011. PMID: 21589885. DOI: 10.1159/000328076

12 Murali R, Soslow RA, and Weigelt B: Classification of endometrial carcinoma: more than two types. Lancet Oncol 15(7): e268-278, 2014. PMID: 24872110. DOI: 10.1016/S1470-2045(13)70591-6

13 Le Gallo M and Bell DW: The emerging genomic landscape of endometrial cancer. Clin Chem 60(1): 98-110, 2014. PMID: 24170611. DOI: 10.1373/clinchem.2013.205740

14 Howitt BE, Shukla SA, Sholl LM, Ritterhouse LL, Watkins JC, Rodig S, Stover E, Strickland KC, D'Andrea AD, Wu CJ, Matulonis UA and Konstantinopoulos PA: Association of 
polymerase e-mutated and microsatellite-instable endometrial cancers with neoantigen load, number of tumor-infiltrating lymphocytes, and expression of PD-1 and PD-L1. JAMA Oncol 1(9): 1319-1323, 2015. PMID: 26181000. DOI: 10.1001/ jamaoncol.2015.2151

15 Brahmer JR, Tykodi SS, Chow LQ, Hwu WJ, Topalian SL, Hwu P, Drake CG, Camacho LH, Kauh J, Odunsi K, Pitot HC, Hamid O, Bhatia S, Martins R, Eaton K, Chen S, Salay TM, Alaparthy S, Grosso JF, Korman AJ, Parker SM, Agrawal S, Goldberg SM, Pardoll DM, Gupta A and Wigginton JM: Safety and activity of anti-PD-L1 antibody in patients with advanced cancer. N Engl J Med 366(26): 2455-2465, 2012. PMID: 22658128. DOI: 10.1056/NEJMoa1200694

16 Topalian SL, Hodi FS, Brahmer JR, Gettinger SN, Smith DC, McDermott DF, Powderly JD, Carvajal RD, Sosman JA, Atkins MB, Leming PD, Spigel DR, Antonia SJ, Horn L, Drake CG, Pardoll DM, Chen L, Sharfman WH, Anders RA, Taube JM, McMiller TL, Xu H, Korman AJ, Jure-Kunkel M, Agrawal S,
McDonald D, Kollia GD, Gupta A, Wigginton JM and Sznol M: Safety, activity, and immune correlates of anti-PD-1 antibody in cancer. N Engl J Med 366(26): 2443-2454, 2012. PMID: 22658127. DOI: 10.1056/NEJMoa1200690

17 Mo Z, Liu J, Zhang Q, Chen Z, Mei J, Liu L, Yang S, Li H, Zhou L and You Z: Expression of PD-1, PD-L1 and PD-L2 is associated with differentiation status and histological type of endometrial cancer. Oncol Lett 12(2): 944-950, 2016. PMID: 27446374. DOI: 10.3892/ol.2016.4744

Received May 23, 2019

Revised June 23, 2019

Accepted June 24, 2019 